Journal of Systems Science and Information

Apr., 2017, Vol. 5, No. 2, pp. 128-147

DOI: $10.21078 /$ JSSI-2017-128-20

\title{
On Consistency in AHP and Fuzzy AHP
}

\author{
Fang LIU \\ School of Mathematics and Information Science, Guangxi University, Nanning 530004, China; School \\ of Management, Xi'an Jiaotong University, Xi'an 710049, China \\ E-mails: fang272@126.com; f_liu@gxu.edu.cn \\ Yanan PENG \\ School of Mathematics and Information Science, Guangxi University, Nanning 530004, China \\ E-mail: 815926672@qq.com \\ Weiguo ZHANG \\ School of Business Administration, South China University of Technology, Guangzhou 510641, China \\ E-mail: wgzhang@scut.edu.cn \\ Witold PEDRYCZ \\ Department of Electrical and Computer Engineering, University of Alberta, Edmonton T6R 2 V4 AB, \\ Canada \\ E-mail: wpedrycz@ualberta.ca
}

\begin{abstract}
The analytic hierarchy process (AHP) is used widely for analyzing decisions made in various real-world applications. Its basic idea is to construct a hierarchy of concepts encountered in a given decision problem and to choose the best alternative according to pairwise comparison matrices given by the decision maker. Under the assumption of fully rational economics, a reasonable decision should be consistent. It becomes an important issue on how to analyze and ensure the consistency of comparison matrices together with the judgments of the decision maker. The main objectives of the present paper are threefold. First, we review the basic idea and methods used to define the consistency and the transitivity of multiplicative reciprocal matrices, additive reciprocal matrices and comparison matrices with fuzzy interval and triangular fuzzy numbers. The existing controversy behind the applications of fuzzy set theory to the AHP in the literature is presented. Second, the consistency of the collective comparison matrices in group decision making based on AHP and fuzzy AHP is further analyzed. We point out that the weak consistency of preference relations with fuzzy numbers in fuzzy AHP and group decision making should be investigated comprehensively. Third, under the consideration of the vagueness in the process of evaluating the judgements, a new concept of fuzzy consistency of comparison matrices in the AHP is given.
\end{abstract}

Keywords decision analysis; AHP; fuzzy set theory; fuzzy consistency; group decision-making

Received July 14, 2016, accepted December 19, 2016

Supported by the National Natural Science Foundation of China (71201037, 71232014, 71571054), China Postdoctoral Science Foundation Funded Project (2014M560794), 2014 Shaanxi Postdoctoral Science Foundation Funded Project, Guangxi Natural Science Foundation (2014GXNSFAA118013), Guangxi Natural Science Foundation for Distinguished Young Scholars (2016GXNSFFA380004) and Science Foundation of Guangxi University for Young Ph.D. (XBZ160257) 


\section{Introduction}

In a real-world life, one is always faced with various choices. With the development of the society and economics, the complexity of the real world is rapidly growing and it is becoming an increasingly demanding task to make the best decisions. Under the assumption of rational behaviour, a feasible method will be helpful to make a reasonable decision. In order to deal with a complex system of decision making, the analytic hierarchy process (AHP) was originally proposed by Saaty ${ }^{[1,2]}$. In the AHP, a decision-making problem is modelled as a hierarchy of criteria, subcriteria and alternatives. The decision maker (DM) may estimate her/his opinions and give relative preferences over a set of alternatives, then form a series of multiplicative reciprocal preference relations. Based on the obtained preference relations, the best alternative is selected. Since it is assumed that the decision behavior is fully rational, the comparison matrices reflecting the decision maker's opinions will be consistent. Consequently, one of the important issues is how to measure and check the consistency of comparison matrices. Saaty ${ }^{[2]}$ proposed the consistency index (C.I.) and the consistency ratio (C.R.) to measure a lack of consistency. It is considered that a consistency ratio of 0.10 or less is acceptable. When a comparison matrix is inconsistent, it is an important issue of how to adjust the inconsistent comparison matrix to the consistent or acceptably consistent one ${ }^{[3]}$.

In a decision process using typical AHP, the goals and the constraints are defined binary and the relative measurements are expressed as numbers located on the scale from 1 to 9 . On the other hand, if one considers that the goals and/or the constraints are not defined clearly, fuzzy set theory may be suitable to be used to analyze the corresponding decision problem ${ }^{[4,5]}$. Moreover, under the consideration of uncertainty existing in the decision maker's opinions, the typical AHP has been extended to a fuzzy version where the relative importance was expressed as a triangular fuzzy number ${ }^{[6,7]}$. Saaty and Vargas ${ }^{[8]}$ also applied an interval of number values to reflect the uncertainty experienced by the decision maker in making pairwise comparisons. Buckley ${ }^{[9]}$ further considered that fuzzy numbers could be used to express an expert's preference and discussed the consistency of the given data. Clearly, when the expert's judgements are expressed as fuzzy numbers, it is more difficult to choose the best alternative and investigate the consistency and acceptable consistency of comparison matrices with fuzzy number entries.

From the above analysis, it is natural that pairwise comparison matrices in the AHP imply consistency of a rational decision. However, the complexity of the real world and the uncertainty of evaluating preference values may lead to the departure from consistency and fuzziness of consistency. In addition, Saaty ${ }^{[10]}$ considered that the consistency and transitivity of the judgements together with the rational behaviors are not assumed previously in the AHP. The main reason may be that the inconsistency degree of the judgements becomes available at the end of the AHP process. The outcome by using the AHP can only reflect the expectations of the decision maker when the decision making problem has been constructed within the hierarchy ${ }^{[10]}$. On the other hand, when a group of experts are invited to make decision, it is an important issue of how to capture the consistency of the group opinions ${ }^{[11]}$. Up to now, a great deal of studies on the consistency and transitivity of various matrices have been published since the book of [2]. Therefore it becomes beneficial to look at the background of the existing methods and clarify the controversies in the literature. We also identify the important issues and focus 
on concepts which are worth studying in the future. The structure of the paper is as follows. Section 2 introduces the ideas and methods used to define the consistency and the transitivity of comparison matrices in the typical AHP. In Section 3, the methods of how to analyze the consistency and the weak transitivity of comparison matrices with fuzzy interval and triangular fuzzy numbers will be analyzed. Section 4 addresses the consistency of the collective preference relations in group decision making based on AHP and fuzzy AHP. The important issues for studying in the future and the new concept for considering the fuzziness of consistency in evaluating the judgements are discussed in Section 5. Finally, Section 6 covers main conclusions.

\section{Consistency in AHP}

In the typical AHP, a practical decision problem is hierarchically structured at different levels where a finite set of alternatives is contained such as $X=\left\{x_{1}, x_{2}, \cdots, x_{n}\right\}$. The decision maker compares each pair of alternatives in $X$ and provides a series of relative assessments to form a preference relation. When the decision maker is perfectly consistent, she/he does not contradict herself/himself and produces a consistent comparison matrix. Then there is an important question of how to define and check the consistency of comparison matrices. Several definitions related to multiplicative reciprocal matrices ${ }^{[2]}$ are given and reviewed in the following subsections.

\subsection{The Consistency Index of Saaty}

In [2], a multiplicative reciprocal preference relation and its consistency are defined as follows:

Definition 1 (see [2]) $A=\left(a_{i j}\right)_{n \times n}$ is called a multiplicative reciprocal preference relation, if the entries of $A$ satisfy the following condition

$$
a_{i j}=\frac{1}{a_{j i}}, \quad a_{i i}=1, \quad a_{i j} \in R^{+}, \quad \forall i, j=1,2, \cdots, n,
$$

where $a_{i j}$ stands for the relative importance of alternatives $x_{i}$ over $x_{j}$.

Definition 2 (see [2]) Let $A=\left(a_{i j}\right)_{n \times n}$ be a multiplicative reciprocal preference relation. If the following condition is satisfied

$$
a_{i j}=a_{i k} a_{k j}, \quad \forall i, j, k=1,2, \cdots, n,
$$

then $A$ is called a consistent multiplicative reciprocal preference relation.

Definition 2 reflects the basic idea that the direct pairwise comparison $a_{i j}$ should be determined exactly by any pair of indirect pairwise comparisons $a_{i k}$ and $a_{k j}(k=1,2, \cdots, n)$ for a consistent comparison matrix. It is based on the ideal assumption that a decision maker is fully rational and exhibits judgements. Obviously, under the consideration of the limitations of human-being to provide this type of numeric quantification and the complexity of a practical decision problem, Definition 2 is idealized and one cannot always generate a consistent preference relation, especially when the number of alternatives is large. Furthermore, Saaty ${ }^{[2]}$ considered that the departure from consistency to some extent is permitted in practical situations. He introduced the so-called consistency index (C.I.) and the consistency ratio (C.R.) to 
measure the derivation level from consistency:

$$
\text { C.I. }=\frac{\lambda_{\max }-n}{n-1}, \quad \text { C.R. }=\frac{\text { C.I. }}{\text { R.I. }}
$$

where $\lambda_{\max }$ and $n$ are the largest eigenvalue and the order of pairwise comparison matrices, respectively. R.I. is a random index dependent on the size of the matrices, and it is the average C.I. of a large number of randomly generated multiplicative preference relations. When C.R. $(A) \leq 0.10, A$ is considered to be acceptably consistent, and for C.I. $(A)=0, A$ is fully consistent. It is seen that there is a threshold of C.R. $(A)=0.10$, which means that the consistency index is ten percent of the mean consistency index of randomly generated matrices. Of much interest is to calculate the thresholds of the largest eigenvalue $\lambda_{\max }^{\mathrm{T}}=n+0.1 \cdot(n-1) \cdot \mathrm{R}$.I. and the relative errors $\varepsilon_{r}=\left(\lambda_{\max }^{\mathrm{T}}-n\right) / n$, which are given in Table 1 . It is seen from Table 1 that with increasing the order $n$, the acceptable relative errors are increasing. Moreover, when C.R. $(A)>0.10$, implying that the comparison matrix is unacceptably consistent, a feasible technique should be used to make it an acceptable one ${ }^{[3]}$. Here according to Table 1 , one can propose a method to adjust the comparison matrix with unacceptable consistency to that with acceptable consistency such that the greatest eigenvalue is equal to $\lambda_{\max }^{\mathrm{T}}$ or less.

Table 1 Thresholds of the largest eigenvalue and the relative errors

\begin{tabular}{ccccccccccccc}
\hline$n$ & 1 & 2 & 3 & 4 & 5 & 6 & 7 & 8 & 9 & 10 & 11 & 12 \\
\hline R.I. & 0 & 0 & 0.52 & 0.89 & 1.12 & 1.26 & 1.36 & 1.41 & 1.46 & 1.49 & 1.52 & 1.54 \\
$\lambda_{\max }^{\mathrm{T}}$ & 0 & 0 & 3.116 & 4.267 & 5.448 & 6.63 & 7.816 & 8.987 & 10.168 & 11.341 & 12.52 & 13.672 \\
$\varepsilon_{r}$ & 0 & 0 & $3.87 \%$ & $6.68 \%$ & $8.96 \%$ & $10.50 \%$ & $11.66 \%$ & $12.34 \%$ & $12.98 \%$ & $13.41 \%$ & $13.82 \%$ & $13.93 \%$ \\
\hline
\end{tabular}

\subsection{Axiomatic Properties of Inconsistency Indices}

In order to estimate the inconsistency level of multiplicative reciprocal comparison matrices, there are various other inconsistency indices presented in the literature such as the geometric consistency index (GCI ${ }^{[12]}$, the index of determinants ${ }^{[13]}$, the harmonic consistency index ${ }^{[14]}$ and others. The basic idea is how to relax the relation in Definition 2 and its variants. Recently, through numerical computations, ten inconsistency indices for multiplicative reciprocal comparison matrices have been surveyed and analyzed in [15]. Here we will not duplicate these inconsistency indices one by one. A remaining question is how to clarify various inconsistency indices and choose a feasible inconsistency index in a decision making process. To answer it, Brunelli and Fedrizzi ${ }^{[16]}$ have given several axiomatic properties to characterize inconsistency indices of multiplicative reciprocal comparison matrices. The given axiomatic framework was further expended and a new axiom was added in [17]. They defined the set of all multiplicative reciprocal comparison matrices as

$$
\mathcal{A}=\left\{\boldsymbol{A}=\left(a_{i j}\right)_{n \times n} \mid a_{i j}>0, a_{i j} a_{j i}=1, \forall i, j, n \geq 2\right\}
$$

and that of consistent ones as

$$
\mathcal{A}^{*}=\left\{\boldsymbol{A}=\left(a_{i j}\right)_{n \times n} \mid \boldsymbol{A} \in \mathcal{A}, a_{i j}=a_{i k} a_{k j}, \forall i, j, k\right\} \subset \mathcal{A} \text {. }
$$


Then an effective inconsistency index is defined as a real-valued function:

$$
I: \mathcal{A} \rightarrow \mathbb{R}
$$

and it should satisfy the following six axiomatic properties:

- Existence of a unique element representing consistency,

- Invariance under permutation of alternatives,

- Monotonicity under reciprocity-preserving mapping,

- Monotonicity on single comparisons,

- Continuity,

- Invariance under inversion of preferences.

It is considered that the inconsistency index satisfying the axioms can be used to correctly evaluate the inconsistency. The obtained results reveal that some of the known inconsistency indices in the literature satisfy these axioms and the others do not. At least, this approach is a valuable attempt to normally characterize the inconsistency indices of multiplicative reciprocal comparison matrices by proposing the axiomatic properties.

One can see that the inconsistency index is reflecting the consistency derivation from the viewpoint of quantity. However, the main objective of decision making is to choose the best alternative, and of much interest is the order of all alternatives. We consider that from the viewpoint of quality, a stable order of alternatives is required. In order to achieve a quantitative ranking of alternatives, the conditions of weak transitivity and weak consistency for the multiplicative reciprocal comparison matrix $A=\left(a_{i j}\right)_{n \times n}$ have been given respectively as ${ }^{[18,19]}$ :

$$
a_{i j}>1, \quad a_{j k}>1 \Rightarrow a_{i k}>1, \quad \forall i, j, k,
$$

and

$$
a_{i j}>1, \quad a_{j k}>1 \Rightarrow a_{i k}>\max \left\{a_{i j}, a_{j k}\right\}, \quad \forall i, j, k .
$$

From the above analysis, if one only wants to chose the best alternative, an inconsistent preference relation should be acceptable under the condition of giving a stable ranking of alternatives.

\section{Consistency in Fuzzy AHP}

In a fuzzy environment, the goals and the constraints presented in a decision making problem can be described in terms of fuzzy sets ${ }^{[4,20]}$. The AHP is a well-known quantitative decision analysis methodology which has been widely used in various practical applications ${ }^{[21]}$. The combination of fuzzy set theory and AHP leads to fuzzy AHP. This extension of the method has attracted much attention in the recent decades. The basic idea is that the relative measurements in AHP are expressed by fuzzy numbers to model the vagueness of the expert's judgements. In what follows, the consistency of several typical preference relations with fuzzy numbers are analyzed in detail. 


\subsection{Additive Reciprocal Preference Relations}

In the AHP, the relative importance $a_{i j}$ is obtained by comparing the alternative $x_{i}$ with the alternative $x_{j}$. The notion of the strength comparison is in agreement with the common way of thinking. However, because of the vagueness and complexity of comparing the alternative $x_{i}$ with the alternative $x_{j}$, the decision maker cannot come up with an exact number to express the relative measurement. Then one may more readily offer the preference degree of the alternative $x_{i}$ over the alternative $x_{j}{ }^{[22]}$. Generally, based on the fuzzy set theory, the binary relation defined on the alternative set $X=\left\{x_{1}, x_{2}, \cdots, x_{n}\right\}$ is defined as

$$
\mu_{R}: X \times X \rightarrow[0,1]
$$

A matrix $B=\left(b_{i j}\right)_{n \times n}$ is determined and the entry $b_{i j}$ is given by

$$
b_{i j}=\mu_{R}\left(x_{i}, x_{j}\right), \quad i, j=1,2, \cdots, n .
$$

If the matrix $B=\left(b_{i j}\right)_{n \times n}$ is further considered as a preference relation, the element $b_{i j}$ stands for the preference degree of the alternative $x_{i}$ over the alternative $x_{j}$ with the reciprocal property expressed as $b_{i j}+b_{j i}=1^{[23]}$. In order to distinguish the multiplicative reciprocal property in (1), it is convenient to recall the preference relation $B=\left(b_{i j}\right)_{n \times n}$ as the additive reciprocal matrix ${ }^{[24]}$ :

Definition 3 (see [23]) $B=\left(b_{i j}\right)_{n \times n} \subset X \times X$ is called as an additive reciprocal matrix, if $b_{i j}$ is the preference intensity of the alternatives $x_{i}$ to $x_{j}$ with $b_{i j}+b_{j i}=1,0 \leq b_{i j} \leq 1$, for $\forall i, j=1,2, \cdots, n$.

In what follows, let us focus on the transitivity and the consistency of additive reciprocal matrices. It is considered that if $x_{i} \succ x_{j}$ and $x_{j} \succ x_{k}$, one should have $x_{i} \succ x_{k}$ with at least the same intensity. The max-min transitivity is defined as ${ }^{[23]}$

$$
b_{i j} \geq \frac{1}{2}, \quad b_{j k} \geq \frac{1}{2} \Rightarrow b_{i k} \geq \min \left\{b_{i j}, b_{j k}\right\}, \quad \forall i, j, k .
$$

The restrict max-min transitivity is a stronger condition and it is further defined as ${ }^{[23]}$

$$
b_{i j} \geq \frac{1}{2}, \quad b_{j k} \geq \frac{1}{2} \Rightarrow b_{i k} \geq \max \left\{b_{i j}, b_{j k}\right\}, \quad \forall i, j, k .
$$

In addition, the additive transitivity is given as

$$
\left(b_{i j}-\frac{1}{2}\right)+\left(b_{j k}-\frac{1}{2}\right)=\left(b_{i k}-\frac{1}{2}\right), \quad \forall i, j, k,
$$

which is stronger than the restrict max-min transitivity and means that the preference degree $b_{i k}$ can be derived indirectly by the preference degrees $b_{i j}$ and $b_{j k}$. Specially, it is assumed that $b_{i j} / b_{j i}$ stands for the comparison ratio of $x_{i}$ over $x_{j}$. From Definition 2, one has the multiplicative transitivity

or

$$
\frac{b_{i j}}{b_{j i}}=\frac{b_{i k}}{b_{k i}} \frac{b_{k j}}{b_{j k}}, \quad \forall i, j, k
$$

$$
b_{i j} b_{j k} b_{k i}=b_{i k} b_{k j} b_{j i}, \quad \forall i, j, k
$$


by considering the case of $b_{i j}=0$, which can be used to give the restrict max-min transitivity ${ }^{[23]}$. For convenience, two definitions of additive reciprocal preference relations with consistency are given as follows ${ }^{[25,26]}$ :

Definition 4 (see [25]) An additive reciprocal matrix $B=\left(b_{i j}\right)_{n \times n}$ is additively consistent, if one has

$$
b_{i j}=b_{i k}-b_{j k}+0.5, \quad \forall i, j, k=1,2, \cdots, n,
$$

where $0<b_{i j}<1$ for $\forall i, j=1,2, \cdots, n$.

Definition 5 (see [25]) An additive reciprocal matrix $B=\left(b_{i j}\right)_{n \times n}$ has the multiplicative consistency, if it satisfies the multiplicative transitivity

$$
b_{i j} b_{j k} b_{k i}=b_{i k} b_{k j} b_{j i}, \quad \forall i, j, k=1,2, \cdots, n,
$$

where $0<b_{i j}<1$ for $\forall i, j=1,2, \cdots, n$.

For the additive consistency in Definition 4 , it is interpreted that if the value of $\left(b_{i j}-\frac{1}{2}\right)$ is considered as the preference intensity of $x_{i}$ over $x_{j}$, the preference intensity of $x_{i}$ over $x_{k}$ should be equal to the sum of the indirect preference intensities of $\left(b_{i j}-\frac{1}{2}\right)$ and $\left(b_{j k}-\frac{1}{2}\right)$, and some equivalent propositions have been given in [25]. Definition 5 is completely based on the typical idea of consistent comparison matrices together with the consistent judgements in Definition 2. It seems that Definition 5 has nothing with the fuzzy set theory except $b_{i j} \in[0,1]$.

\subsection{Interval Multiplicative Reciprocal Matrices}

To model the uncertainty presented in the pairwise comparisons, the decision maker may give an interval of numerical values to estimate her/his judgements. This results in an interval comparison matrix ${ }^{[8]}$. It is seen that a real interval $[a, b]$ can be treated as a fuzzy number with the membership function ${ }^{[27]}$

$$
\mu: \mathbb{R} \rightarrow[0,1]
$$

and

$$
\mu(x)= \begin{cases}1, & x \in[a, b], \\ 0, & \text { otherwise }\end{cases}
$$

It is convenient to recall the interval comparison matrices as interval multiplicative preference relations to clarify various terminologies used in the literature [24]. The following definition is introduced:

Definition 6 (see [8]) An interval multiplicative reciprocal matrix $\tilde{A}$ is defined as

$$
\tilde{A}=\left(\tilde{a}_{i j}\right)_{n \times n}=\left[\begin{array}{cccc}
{[1,1]} & {\left[a_{12}^{-}, a_{12}^{+}\right]} & \cdots & {\left[a_{1 n}^{-}, a_{1 n}^{+}\right]} \\
{\left[a_{21}^{-}, a_{21}^{+}\right]} & {[1,1]} & \cdots & {\left[a_{2 n}^{-}, a_{2 n}^{+}\right]} \\
\vdots & \vdots & \ddots & \vdots \\
{\left[a_{n 1}^{-}, a_{n 1}^{+}\right]} & {\left[a_{n 2}^{-}, a_{n 2}^{+}\right]} & \cdots & {[1,1]}
\end{array}\right],
$$

where $a_{i j}^{-}$and $a_{i j}^{+}$are non-negative real numbers, $a_{i j}^{-} \leq a_{i j}^{+}, a_{i j}^{-}=1 / a_{j i}^{+}$and $a_{i j}^{+}=1 / a_{j i}^{-} . \tilde{a}_{i j}$ indicates that the alternative $x_{i}$ is between $a_{i j}^{-}$and $a_{i j}^{+}$times as important as the alternative $x_{j}$. 
For the consistency analysis of $\tilde{A}$, Wang, et al. ${ }^{[28]}$ have considered that an interval multiplicative reciprocal matrix is consistent, if there exists a consistent matrix with elements lying in the entries of $\tilde{A}$. The corresponding definition is given as follows:

Definition 7 (see [28]) An interval multiplicative reciprocal matrix $\tilde{A}$ is consistent, if the convex feasible region $S_{w}=\left\{w=\left(w_{1}, w_{2}, \cdots, w_{n}\right) \mid a_{i j}^{-} \leq w_{i} / w_{j} \leq a_{i j}^{+}, \sum_{i=1}^{n} w_{i}=1, w_{i}>0, \forall i\right\}$ is nonempty.

It is seen that Definition 7 is directly used the consistency definition of multiplicative reciprocal matrices in Definition 2. The reciprocity and the transitivity of fuzzy intervals have been completely ignored. However, as pointed out in [29], the reciprocal condition $\tilde{a}_{i j}=1 / \tilde{a}_{j i}$ does not mean $\tilde{a}_{i j} \cdot \tilde{a}_{j i}=1$ and the transitivity property of all entries does not hold in the form $\tilde{a}_{i j}=\tilde{a}_{i k} \cdot \tilde{a}_{k j}$. It implies that the consistency of interval multiplicative reciprocal matrices cannot be defined as that in Definition 2. In other words, the consistency condition in Definition 2 is incompatible with the operation law of fuzzy interval in fuzzy set theory ${ }^{[30]}$.

Furthermore, the reciprocal property of preference relation is one of the axioms as the foundation of the AHP in [10]. One can observe from Definition 6 that the decision maker must consider the reciprocal property $a_{i j}^{-}=1 / a_{j i}^{+}$and $a_{i j}^{+}=1 / a_{j i}^{-}$. Then Liu ${ }^{[31]}$ has investigated the consistency and acceptable consistency of $\tilde{A}$. That is, letting $C=\left(c_{i j}\right)_{n \times n}$ and $D=\left(d_{i j}\right)_{n \times n}$ where

$$
c_{i j}=\left\{\begin{array}{cc}
a_{i j}^{+}, & i<j, \\
1, & i=j, \\
a_{i j}^{-}, & i>j,
\end{array} \quad d_{i j}=\left\{\begin{array}{cc}
a_{i j}^{-}, & i<j, \\
1, & i=j, \\
a_{i j}^{+}, & i>j,
\end{array}\right.\right.
$$

one has the two definitions:

Definition 8 (see [31]) An interval multiplicative reciprocal preference relation $\tilde{A}$ is said to be consistent, if the multiplicative preference relations $C$ and $D$ determined by using (19) are all consistent.

Definition 9 (see [31]) If the multiplicative reciprocal preference relations $C$ and $D$ determined by utilizing (19) are all of acceptable consistency, then $\tilde{A}$ is acceptably consistent. Otherwise, $U$ is said to be unacceptably consistent.

From Definitions 8 and 9, the conditions of consistency and acceptable consistency can be easily checked by using the idea given in Definition 2. But Definitions 8 and 9 are based on two multiplicative reciprocal matrices and the transitivity of fuzzy intervals is still not considered. Recently, Wang ${ }^{[32]}$ has pointed out that Definition 8 is technically incorrect by using a numerical example. The reason is based on the fact that interval multiplicative reciprocal matrices may be consistent or inconsistent by only changing the comparison orders of two alternatives. It is considered that the consistency of preference relations should keep the invariance with respect to permutations of decision alternatives. We should confirm that for the typical consistency in the AHP, the invariance of consistency with respect to permutations of the alternatives should be holden due to the reciprocal property $a_{i j}=1 / a_{j i}$. However, when the judgments of the decision maker are expressed by using fuzzy numbers, one should ask if the invariance of consistency with respect to permutations of the alternatives is still holden. This important 
problem should be answered and studied in the future.

Furthermore, Wang ${ }^{[32]}$ gave a new definition of consistent interval multiplicative reciprocal matrices to correct Definition 8 as

Definition 10 (see [32]) An interval multiplicative reciprocal matrix $\tilde{A}$ is geometrically consistent, if the following transitive condition is satisfied

$$
a_{i j}^{-} a_{i j}^{+}=a_{i k}^{-} a_{i k}^{+} a_{k j}^{-} a_{k j}^{+}, \quad i, j, k=1,2, \cdots, n .
$$

Clearly, if one has the following transitive conditions

$$
a_{i j}^{-}=a_{i k}^{-} a_{k j}^{-}, \quad a_{i j}^{+}=a_{i k}^{+} a_{k j}^{+}, \quad i, j, k=1,2, \cdots, n,
$$

the interval multiplicative reciprocal matrix $\tilde{A}$ is consistent in terms of Definition 10. Consequently, Definition 10 is an extension of Definition 2 by considering the transitivity of left bounds and right bounds of interval entries in $\tilde{A}$. Moreover, from the process of giving comparison matrices, one should consider the reciprocity of $a_{i j}^{-}=1 / a_{j i}^{+}$and $a_{i j}^{+}=1 / a_{j i}^{-}$. Under the consideration of the reciprocity, Eq. (20) can be rewritten in the form

$$
a_{i j}^{+} a_{j k}^{+} a_{k i}^{+}=a_{i k}^{+} a_{k j}^{+} a_{j i}^{+}, \quad \forall i, j, k=1, \cdots, n
$$

or

$$
a_{i j}^{-} a_{j k}^{-} a_{k i}^{-}=a_{i k}^{-} a_{k j}^{-} a_{j i}^{-}, \quad \forall i, j, k=1,2, \cdots, n .
$$

It is noted that the transitive conditions in (22) and (23) are identical with that given in Definition 5 for an additive reciprocal matrix with multiplicative consistency. That is to say, the consistency condition in Definition (10) is only a rewritten version of the known ones. In addition, Eqs. (22) and (23) require the left bounds or the right bounds of interval entries in $\tilde{A}$ to satisfy the transitive conditions. It is hard to understand from the viewpoint of giving comparison ratios in a decision making process. Definition 10 does not reflect the reciprocal property and the idea of fuzzy set theory. The requirement of the transitive conditions in (20) seems mainly a mathematical technique to some extent in order to make the definition have the invariance with respect to permutations of alternatives. The basic idea of Definition 10 is to characterize the consistency of interval multiplicative reciprocal matrices by using a special multiplicative reciprocal preference relation in (20). The effectiveness of Definition 10 is in doubt and it should be further investigated in the future.

It is noted that Xia and Chen ${ }^{[33]}$ have defined the weak transitivity of interval multiplicative reciprocal preference relations by using a possibility degree formula to compare two interval multiplicative reciprocal preference values, namely

Definition 11 (see [33]) An interval multiplicative reciprocal matrix $\tilde{A}$ is weakly transitive if application of $P_{\tilde{a}_{i j} \succ[1,1]} \geq 0.5$ and $P_{\tilde{a}_{j k} \succ[1,1]} \geq 0.5$ leads to $P_{\tilde{a}_{i k} \succ[1,1]} \geq 0.5$ for $i, j, k=1,2, \cdots, n$, where $P_{\bullet}$ denotes the possibility degree.

Furthermore, Xia and Chen ${ }^{[33]}$ have given the definition of consistent interval multiplicative reciprocal matrices, namely

Definition 12 (see [33]) An interval multiplicative reciprocal matrix $\tilde{A}$ is consistent if one has

$$
\frac{\tilde{a}_{i j}}{\tilde{a}_{i k} \tilde{a}_{k j}}=\frac{\tilde{a}_{i k} \tilde{a}_{k j}}{\tilde{a}_{i j}}
$$


In virtue of the laws of fuzzy numbers, application of (24) yields

$$
\left[\frac{a_{i j}^{-}}{a_{i k}^{+} a_{k j}^{+}}, \frac{a_{i j}^{+}}{a_{i k}^{-} a_{k j}^{-}}\right]=\left[\frac{a_{i k}^{-} a_{k j}^{-}}{a_{i j}^{+}}, \frac{a_{i k}^{+} a_{k j}^{+}}{a_{i j}^{-}}\right] .
$$

Then according to Lemma 1 in [33], we can further obtain the transitive condition (20). As compared to the studies of consistency in the literature, one can see that the studies of the weak transitivity of interval multiplicative reciprocal matrices in [33] is a feasible attempt and it is worthy of investigating and extending widely.

\subsection{Other Comparison Matrices with Fuzzy Numbers}

Except for interval multiplicative reciprocal matrices, the decision maker may express her/his judgements by using interval additive reciprocal matrices, triangular fuzzy reciprocal matrices. First, let us give the definition of interval additive reciprocal matrices as follows ${ }^{[34]}$.

Definition 13 (see [34]) An interval additive reciprocal matrix $\tilde{B}$ is expressed as

$$
\tilde{B}=\left(\tilde{b}_{i j}\right)_{n \times n}=\left[\begin{array}{cccc}
{[0.5,0.5]} & {\left[b_{12}^{-}, b_{12}^{+}\right]} & \cdots & {\left[b_{1 n}^{-}, b_{1 n}^{+}\right]} \\
{\left[b_{21}^{-}, b_{21}^{+}\right]} & {[0.5,0.5]} & \cdots & {\left[b_{2 n}^{-}, b_{2 n}^{+}\right]} \\
\vdots & \vdots & \ddots & \vdots \\
{\left[b_{n 1}^{-}, b_{n 1}^{+}\right]} & {\left[b_{n 2}^{-}, b_{n 2}^{+}\right]} & \cdots & {[0.5,0.5]}
\end{array}\right],
$$

where $b_{i j}^{-}$and $b_{i j}^{+}$are non-negative real numbers, $b_{i j}^{-} \leq b_{i j}^{+}, b_{i j}^{-}+b_{j i}^{+}=1$ for all $1 \leq i, j \leq n$. $\tilde{b}_{i j}$ indicates the importance degree of alternative $x_{i}$ over $x_{j}$. Especially, under the consideration of the multiplicative consistency of additive reciprocal matrix, we have $0<b_{i j}^{-} \leq b_{i j}^{+}<1$.

Two definitions of consistent interval additive reciprocal matrices are further presented as follows $^{[26,34]}$.

Definition 14 (see [26]) An interval additive reciprocal matrix $\tilde{B}$ is additively consistent, if there is a vector $w=\left(w_{1}, w_{2}, \cdots, w_{n}\right)$ such that

$$
b_{i j}^{-} \leq 0.5\left(w_{i}-w_{j}+1\right) \leq b_{i j}^{+}, \quad i, j=1,2, \cdots, n .
$$

Definition 15 (see [34]) An interval additive reciprocal matrix $\tilde{B}$ is multiplicative consistent, if there exists a vector $w=\left(w_{1}, w_{2}, \cdots, w_{n}\right)$ such that

$$
b_{i j}^{-} \leq \frac{w_{i}}{w_{i}+w_{j}} \leq b_{i j}^{+}, \quad i, j=1,2, \cdots, n .
$$

It is obvious that Definitions 14 and 15 are only given through a direct application of Definitions 4 and 5 , respectively.

Liu, et al. ${ }^{[24,35]}$ have considered the additive reciprocity of $b_{i j}^{-}+b_{j i}^{+}=1$ and defined consistent interval additive reciprocal matrices. Letting $P=\left(p_{i j}\right)_{n \times n}$ and $Q=\left(q_{i j}\right)_{n \times n}$ be expressed as

$$
p_{i j}=\left\{\begin{array}{cc}
b_{i j}^{+}, & i<j, \\
0.5, & i=j, \\
b_{i j}^{-}, & i>j,
\end{array} \quad q_{i j}=\left\{\begin{array}{cc}
b_{i j}^{-}, & i<j, \\
0.5, & i=j, \\
b_{i j}^{+}, & i>j .
\end{array}\right.\right.
$$

The following definitions are given: 
Definition 16 (see [35]) An interval additive reciprocal matrix $\tilde{B}$ is additively consistent, if $P=\left(p_{i j}\right)_{n \times n}$ and $Q=\left(q_{i j}\right)_{n \times n}$ are additively consistent.

Definition 17 (see [24]) An interval additive reciprocal matrix $\tilde{B}$ is multiplicative consistent, if $P=\left(p_{i j}\right)_{n \times n}$ and $Q=\left(q_{i j}\right)_{n \times n}$ are multiplicative consistent.

An interval additive reciprocal matrix $\tilde{B}$ with the conditions in Definitions 16 and 17 is also consistent by using Definitions 14 and 15, respectively. These definitions are not related to the comparison of interval additive reciprocal preference values. Basically, one should consider the fuzzy relation of interval numbers and study the transitivity of interval additive reciprocal matrices in the future.

On the other hand, based on the operation laws of interval fuzzy number, Wang and $\mathrm{Li}^{[}{ }^{[36]}$ extended the definition of consistent additive reciprocal matrices to that of consistent interval additive reciprocal matrices. They obtained the following two definitions:

Definition 18 (see [36]) An interval additive reciprocal matrix $\tilde{B}$ is called to be additively consistent, if the following additive transitivity holds:

$$
\tilde{b}_{i j} \oplus \tilde{b}_{j k} \oplus \tilde{b}_{k i}=\tilde{b}_{j i} \oplus \tilde{b}_{k j} \oplus \tilde{b}_{i k}, \quad i, j, k=1,2,3, \cdots, n .
$$

Definition 19 (see [36]) An interval additive reciprocal matrix $\tilde{B}$ is called multiplicative consistent, if one has the following multiplicative transitivity:

$$
\frac{\tilde{b}_{i j}}{\tilde{b}_{j i}} \otimes \frac{\tilde{b}_{j k}}{\tilde{b}_{k j}} \otimes \frac{\tilde{b}_{k i}}{\tilde{b}_{i k}}=\frac{\tilde{b}_{j i}}{\tilde{b}_{i j}} \otimes \frac{\tilde{b}_{k j}}{\tilde{b}_{j k}} \otimes \frac{\tilde{b}_{i k}}{\tilde{b}_{k i}}, \quad i, j, k=1,2, \cdots, n .
$$

If one considers that the equal sign " $="$ in (28) and (29) implies two fully identical interval number, it yields ${ }^{[37]}$

$$
\begin{aligned}
& b_{i j}^{-}+b_{j k}^{-}+b_{k i}^{-}=b_{j i}^{-}+b_{k j}^{-}+b_{i k}^{-}, \quad b_{i j}^{+}+b_{j k}^{+}+b_{k i}^{+}=b_{j i}^{+}+b_{k j}^{+}+b_{i k}^{+}, \\
& \frac{b_{i j}^{-}}{b_{j i}^{-}} \frac{b_{j k}^{-}}{b_{k j}^{-}} \frac{b_{k i}^{-}}{b_{i k}^{-}}=\frac{b_{j i}^{-}}{b_{i j}^{-}} \frac{b_{k j}^{-}}{b_{j k}^{-}} \frac{b_{i k}^{-}}{b_{k i}^{-}}, \quad \frac{b_{i j}^{+}}{b_{j i}^{+}} \frac{b_{j k}^{+}}{b_{k j}^{+}} \frac{b_{k i}^{+}}{b_{i k}^{+}}=\frac{b_{j i}^{+}}{b_{i j}^{+}} \frac{b_{k j}^{+}}{b_{j k}^{+}} \frac{b_{i k}^{+}}{b_{k i}^{+}}
\end{aligned}
$$

That is, the conditions in Definitions 18 and 19 require that two numeric matrices $\left(b_{i j}^{-}\right)_{n \times n}$ and $\left(b_{i j}^{+}\right)_{n \times n}$ are additive consistent and multiplicative consistent respectively. The requirements are much stricter than those given in Definitions 14 and 15 and they have nothing to do with the transitivity of interval numbers.

In addition, the consistency and transitivity of triangular and trapezoidal fuzzy comparison matrices have been also investigated in the literature. For example, Buckley ${ }^{\left[{ }^{[}\right]}$has directly extended the consistency definition of multiplicative reciprocal matrices in [2] and introduced the following definition:

Definition 20 (see [9]) A fuzzy positive reciprocal matrix $\tilde{A}=\left(\tilde{a}_{i j}\right)_{n \times n}$ is consistent if and only if $\tilde{a}_{i j} \approx \tilde{a}_{i k} \tilde{a}_{k j}$, where $\tilde{a}_{i j}$ denote trapezoidal fuzzy numbers.

Based on Definition 20 and the operation laws of fuzzy numbers ${ }^{[30]}$, it is the key of how to understand and define the fuzzy relation of " $\approx$ ". In [9], for two fuzzy numbers $\tilde{M}$ and $\tilde{N}$ with the membership functions $\mu_{m}(x)$ and $\mu_{n}(x)$ respectively, one defines a comparison function as

$$
v(\tilde{M} \geq \tilde{N})=\sup _{x \geq y}\left(\min \left(\mu_{m}(x), \mu_{n}(x)\right)\right) .
$$


When $v(\tilde{M} \geq \tilde{N})=1$ and $v(\tilde{N} \geq) \tilde{M}<\theta$, where $\theta$ is some fixed positive fraction less or equal to one, it means that $\tilde{M}$ is greater than $\tilde{N}$. When $\min (v(\tilde{M} \geq \tilde{N}), v(\tilde{M} \leq \tilde{N})) \geq \theta$, one says that $\tilde{M}$ is approximately equal to $\tilde{N}$, namely $\tilde{M} \approx \tilde{N}$. In form, Definition 20 is a direct extension of consistent multiplicative reciprocal comparison matrices ${ }^{[9]}$. On the other hand, Wang and Chen ${ }^{[38]}$ considered that Definition 20 is suitable for characterizing the consistency of triangular fuzzy reciprocal preference relations. However, the method of extending directly the typical definition for consistent multiplicative reciprocal matrices ${ }^{[2]}$ is not suitable for consistent comparison matrices with fuzzy numbers ${ }^{[29]}$. The reciprocal property of preference relations in $\mathrm{AHP}^{[10]}$ and fuzzy AHP is a basic requirement and it should be considered ${ }^{[39]}$. Under the consideration of the reciprocal property in triangular fuzzy reciprocal matrices, Liu, et al. ${ }^{[40]}$ gave the corresponding consistency definition and pointed out the existing shortcomings of the propositions given in [38]. The triangular fuzzy reciprocal preference relation is given as:

Definition 21 (see [7]) A triangular fuzzy reciprocal preference relation is represented as

$$
\tilde{T}=\left(\tilde{t}_{i j}\right)_{n \times n}=\left[\begin{array}{cccc}
(1,1,1) & \left(l_{12}, m_{12}, u_{12}\right) & \cdots & \left(l_{1 n}, m_{1 n}, u_{1 n}\right) \\
\left(l_{21}, m_{21}, u_{21}\right) & (1,1,1) & \cdots & \left(l_{2 n}, m_{2 n}, u_{2 n}\right) \\
\vdots & \vdots & \ddots & \vdots \\
\left(l_{n 1}, m_{n 1}, u_{n 1}\right) & \left(l_{n 2}, m_{n 2}, u_{n 2}\right) & \cdots & (1,1,1)
\end{array}\right],
$$

where $\tilde{t}_{i j}$ stands for the triangular fuzzy degree of the alternative (criterion) $x_{i}$ over $x_{j} . l_{i j}$ and $u_{i j}$ represent the lower and upper bounds of the triangular fuzzy number $\tilde{t}_{i j}$, respectively and $m_{i j}$ is the median value. $l_{i j}, m_{i j}$ and $u_{i j}$ are non-negative real numbers with $l_{i j} \leq m_{i j} \leq u_{i j}$ and $l_{i j} u_{j i}=m_{i j} m_{j i}=u_{i j} l_{j i}=1$.

Then three preference relations $T^{L}, T^{M}$ and $T^{R}$ are constructed from $\tilde{T}=\left(\left(l_{i j}, m_{i j}, u_{i j}\right)\right)_{n \times n}$ and let $T^{L}=\left(t_{i j}^{L}\right)_{n \times n}, T^{M}=\left(t_{i j}^{M}\right)_{n \times n}$ and $T^{R}=\left(t_{i j}^{R}\right)_{n \times n}$, where

$$
t_{i j}^{L}=\left\{\begin{array}{cc}
l_{i j}, & i<j, \\
1, & i=j, \\
u_{i j}, & i>j,
\end{array} \quad t_{i j}^{R}=\left\{\begin{array}{cc}
u_{i j}, & i<j, \\
1, & i=j, \\
l_{i j}, & i>j,
\end{array}\right.\right.
$$

and $t_{i j}^{M}=m_{i j}, \forall i, j=1,2, \cdots, n$. One further has the following definition:

Definition 22 (see [40]) If three reciprocal preference relations $T^{L}, T^{M}$ and $T^{R}$ are consistent, $\tilde{T}$ is said to be a consistent triangular fuzzy reciprocal preference relation. Otherwise, $\tilde{T}$ is said to be inconsistent.

Unfortunately, similar to Definition 8, Wang ${ }^{[41]}$ also stated that Definition 22 is not ideal and there exists the technical shortcoming. That is, Definition 22 is dependent on the permutations of alternatives. A definition similar to Definition 10 is given as:

Definition 23 (see [41]) $\tilde{T}$ is said to be a consistent triangular fuzzy reciprocal preference relation, if one has

$$
\tilde{t}_{i j} \otimes \tilde{t}_{j k} \otimes \tilde{t}_{k i}=\tilde{t}_{i k} \otimes \tilde{t}_{k j} \otimes \tilde{t}_{j i}
$$

for $i, j, k=1,2, \cdots, n$. 
Clearly, Definition 23 can be analyzed similar to Definition 10 and it is still unsatisfactory. According to the above analysis, it is difficult to define and test the consistency of preference relations with fuzzy numbers. It is found that there is a substantial controversy in analyzing the consistency of interval and triangular fuzzy multiplicative reciprocal matrices and this may be clarified in the coming works.

\section{Consistency in Group Decision Making}

In real-life applications, a decision may be reached by a group of experts or members. It is an important question on how to aggregate the preference information of individuals and arrive at a consensus. It is also noted that many operators for aggregating information have been surveyed in [42]. In what follows, several typical aggregation methods of individual information and the consistency of group judgements are focused when the AHP and the fuzzy AHP are applied in group decision-making.

\subsection{The Geometric Mean Method}

Under the assumption that a decision is determined by voting of a committee, Black ${ }^{[11]}$ developed a technique to solve group decision-making problem where each member of the committee ranked the alternatives in a definite order of preference. Moreover, it is seen that the analytic hierarchy process has been successfully applied to various group decision-making problems ${ }^{[43]}$. The geometric mean is considered as the uniquely appropriate rule for aggregating the individual judgements in the AHP. The basic reason is that the geometric mean method can preserve the reciprocal property in the combined pairwise comparison matrix. Another important and interesting issue is the consistency of the combined pairwise comparison matrix and the contributions of an individual to the group judgements. It is assumed that a decision making problem has a finite set of alternatives $X=\left\{x_{1}, x_{2}, \cdots, x_{n}\right\}$. The group of decision makers is denoted by $\left\{e_{1}, e_{2}, \cdots, e_{m}\right\}$ and the corresponding weight vector is $v=\left\{v_{1}, v_{2}, \cdots, v_{m}\right\}$ with $\sum_{k=1}^{m} v_{k}=1, v_{k} \geq 0$. The $k$ th decision maker gives his/her preference information as a multiplicative reciprocal preference relation $A_{k}=\left(a_{i j}^{k}\right)_{n \times n}$. Generally the geometric mean method can be extended to the weighted geometric mean method, which can be used to aggregate the individual multiplicative reciprocal preference relations to a collective one $A_{c}=\left(\prod_{k=1}^{m}\left(a_{i j}^{k}\right)^{v_{k}}\right)_{n \times n}$. Then one has the following theorem.

Theorem 1 (see [44]) Let $\lambda_{\max }^{k}$ be the greatest eigenvalue of the multiplicative reciprocal preference relation $A^{k}=\left(a_{i j}^{k}\right)_{n \times n}, k=1,2, \cdots, m$. It is supposed that $A_{c}=\left(a_{i j}^{c}\right)_{n \times n}=$ $\left(\prod_{k=1}^{m}\left(a_{i j}^{k}\right)^{v_{k}}\right)_{n \times n}$ with $\sum_{k=1}^{m} v_{k}=1, v_{k} \geq 0$, and $\lambda_{\max }^{A_{c}}$ is the greatest eigenvalue of $A_{c}$. One has

$$
\lambda_{\max }^{A_{c}} \leq \max \left\{\lambda_{\max }^{1}, \lambda_{\max }^{2}, \cdots, \lambda_{\max }^{m}\right\}
$$

It is seen from Theorem 1 that the collective multiplicative reciprocal matrix $A_{c}$ can be confirmed to be acceptably consistent when all the individual ones are acceptably consistent by using the consistency ratio of Saaty ${ }^{[2]}$. Although the above result has been given in [45], the proof was shown to be wrong by using a counter example in [46]. In particular, when the individual multiplicative reciprocal matrices are consistent, the collective one obtained by using the geometric mean method is consistent. Moreover, based on Definition 9, when 
individual interval multiplicative reciprocal matrices are all acceptably consistent, the collective one obtained by using the induced weighted geometric average (IWGA) operator is acceptably consistent $^{[44]}$.

\subsection{The Ordered Weighted Averaging (OWA) Operators}

The ordered weighted averaging (OWA) operators are a family of aggregation methods of information with a series of parameters ${ }^{[47]}$. The maximum, minimum and the simple average operators are the special cases of the OWA operators ${ }^{[48]}$. An OWA operator of dimensionality $n$ was first defined in [49] as

Definition 24 (see [49]) A mapping $F:[0,1]^{n} \rightarrow[0,1]$ is called an OWA operator of dimension $n$ with the associated weight vector $w=\left(w_{1}, w_{2}, \cdots, w_{n}\right)\left(w_{i} \in(0,1), \sum_{i=1}^{n} w_{i}=1\right)$ such that

$$
F\left(a_{1}, a_{2}, \cdots, a_{n}\right)=w_{1} b_{1}+w_{2} b_{2}+\cdots+w_{n} b_{n},
$$

where $b_{j}$ is the $j$ th largest element in the collection $a_{1}, a_{2}, \cdots, a_{n}$.

In the OWA operators, a lot of importance is paid to the determination of the associated weight vector ${ }^{[48,50]}$. Yager ${ }^{[49]}$ stated that there are at least two approaches: Applying some kind of learning mechanism and giving the meaning of the weights $w_{i}$. One of the methods for obtaining the weighting vector is to associate the OWA aggregation with a linguistic quantifier $Q$, which can be represented as a fuzzy subset on the unit interval.

Furthermore, Yager and Filev ${ }^{[51]}$ proposed the induced ordered weighted averaging (IOWA) Operator, which is a more general type of OWA operator and given as follows:

Definition 25 (see [51]) An IOWA operator of dimension $m$ is a function $\Phi_{w}:(\Re \times \Re)^{n} \rightarrow$ $\Re$ such that

$$
\Phi_{w}\left(\left\langle\mu_{1}, p_{1}\right\rangle, \cdots,\left\langle\mu_{n}, p_{n}\right\rangle\right)=\sum_{i=1}^{n} w_{i} \cdot p_{\sigma(i)} .
$$

Here the associated weight vector is $w=\left(w_{1}, \cdots, w_{n}\right)$ with $w_{i} \in[0,1]$ and $\sum_{i=1}^{n} w_{i}=1$. $\left\langle\mu_{\sigma(i)}, p_{\sigma(i)}\right\rangle$ is the two-tuple with the $i$ th highest value $\mu_{\sigma(i)}$ in the set $\left\{\mu_{1}, \mu_{2}, \cdots, \mu_{n}\right\} . \sigma$ is a permutation of $\{1,2, \cdots, n\}$ such that $\mu_{\sigma(i)} \geq \mu_{\sigma(i+1)}, \forall i=1,2, \cdots, n-1$.

It is obvious that the OWA and the IOWA operators are feasible to aggregate the information expressed by additive reciprocal preference relations in group decision making ${ }^{[52,53]}$. Then the consistency and the transitivity of the collective additive reciprocal matrix should be investigated. When individual additive reciprocal matrices are all additive consistent according to Definition 4, we can affirm that the collective additive reciprocal matrix by using the OWA and the IOWA operators is additively consistent. But when individual additive reciprocal matrices are all multiplicative consistent according to Definition 5, one cannot assure that the collective additive reciprocal matrix by using the OWA and the IOWA operators is multiplicative consistent. The main reason is that the additive consistency is archived by using the linear addition method and the multiplicative consistency is reached by using the multiplication method. For the weak transitivity of the collective additive reciprocal matrix, we cannot find any result in the literature, and this topic should be studied in the future. In addition, the IOWA operators can be used to aggregate interval additive reciprocal preference relations and the corresponding group decision making problems have been considered in [54]. 


\subsection{The Ordered Weighted Geometric (OWG) Operators}

Moreover, in order to aggregate the information represented by multiplicative reciprocal preference relations, the ordered weighted geometric (OWG) operator was given as follows:

Definition 26 (see $[55,56]$ ) An OWG operator of dimension $n$ is a mapping $\phi^{G}: R^{n} \rightarrow R$ such that

$$
\phi^{G}\left(a_{1}, a_{2}, \cdots, a_{n}\right)=\prod_{k=1}^{n} b_{k}^{w_{k}},
$$

where $w=\left(w_{1}, w_{2}, \cdots, w_{n}\right)\left(w_{i} \in(0,1), \sum_{i=1}^{n} w_{i}=1\right)$ is the associated weight vector and $b_{j}$ is the $j$ th largest element in the collection $a_{1}, a_{2}, \cdots, a_{n}$.

The methods of determining the associated weight vector $w$ are similar to those given in [49]. The induced ordered weighted geometric (IOWG) operator is further defined as follows:

Definition 27 (see [57]) An IOWG operator of dimension $n$ is a mapping $\phi_{w}^{G}: R^{n} \rightarrow R$ such that

$$
\phi_{w}^{G}\left(\left\langle u_{1}, a_{1}\right\rangle,\left\langle u_{2}, a_{2}\right\rangle, \cdots,\left\langle u_{n}, a_{n}\right\rangle\right)=\prod_{k=1}^{n} b_{k}^{w_{k}},
$$

where $w=\left(w_{1}, w_{2}, \cdots, w_{n}\right)\left(w_{i} \in(0,1), \sum_{i=1}^{n} w_{i}=1\right)$ is the associated weight vector and $b_{j}$ is the $j$ th largest element in the collection $a_{1}, a_{2}, \cdots, a_{n} .\left\langle u_{\sigma(i)}, a_{\sigma(i)}\right\rangle$ is the two-tuple with the $i$ th highest value $u_{\sigma(i)}$ in the set $\left\{u_{1}, u_{2}, \cdots, u_{n}\right\} . \sigma$ is a permutation of $\{1,2, \cdots, n\}$ such that $u_{\sigma(i)} \geq u_{\sigma(i+1)}, \forall i=1,2, \cdots, n-1$.

When the associated weight vector $w=(1 / n, 1 / n, \cdots, 1 / n)$, the OWG and the IOWG operators are reduced to the geometric mean $(\mathrm{GM})$ operator ${ }^{[57]}$. Similar to those in applying the OWA and IOWA operators, once a method of determining the associated weight vector $w$ is given, one has the corresponding OWG and IOWG operators. In virtue of Theorem 1, it is found that the collective multiplicative reciprocal matrix by using the OWG and IOWG operators is consistent or acceptably consistent when all individual ones are consistent or acceptably consistent. On the other hand, applying the OWG operator or the IOWG operator to aggregate interval multiplicative reciprocal matrices, the similar results can be obtained if Definition 9 is used to characterize the acceptable consistency of interval multiplicative reciprocal preference relations ${ }^{[24]}$. Specially, it should be pointed out that the formula of obtaining the associated exponential weight vector in [24] is only suitable in the case that the consistency indexes are lower than 1. For the case that the applied consistency-index values are higher than 1 , the normalized method as the one in [58] can be used to modify the proposed formula in [24]. In the end, it is concluded that the consistency of the group is dependent on the consistency of each individual decision maker, and the inconsistency indexes of the collective preference relations may be dependent on the permutations of alternatives ${ }^{[24,58]}$. When an individual decision maker is in extremely contrary to the most decision makers in the group, it should be considered carefully how to get the consensus and achieve a reasonable decision.

\section{Discussions}

From the above analysis, one can see that when one introduces the fuzzy set theory into the analytic hierarchy process, the complexity of decision analysis is increasing. One of the reasons 
is that it is difficult to capture the consistency and the transitivity of preference relations with fuzzy numbers. Saaty and Tran ${ }^{[59]}$ have stated that the fuzzy set theory may be invalid in the AHP by fuzzifying judgements. Ramík and Korviny ${ }^{[60]}$ noted that the AHP is a special case of the fuzzy model, and the fuzzy model cannot be worse than the crisp model. This urges us to investigate the compatibility of the idea in fuzzy set theory and the consistency of the decision maker's judgements. One can see that the consistency of multiplicative reciprocal preference relations in the AHP means the cardinal transitivity in the strength of preferences. It represents the idea that the comparison ratio of two alternatives determined by using a direct comparison should be equal to that obtained by using an indirect comparison of a chain of alternatives. In the typical AHP, since an exact number is applied to evaluate the judgement of the decision maker, the equality of two comparison ratios by using different procedures can be reached for a perfectly rational case. However, when the fuzzy set theory is applied to the AHP, the judgements of the decision maker are no longer exact and they are with fuzzy uncertainty. Since the judgements of the decision maker are vague, it seems unreasoning and meaningless to require the strict equality of two fuzzifying judgements obtained by using direct and indirect comparisons respectively. That is, the restrict requirement of consistency is incompatible with the idea of fuzzy set theory to model the vagueness in giving pairwise comparisons. As shown in [22], in a real life, the transitivity may not be an inherent property of fuzzy preferences and a more general class of reflexive fuzzy relations seems reasonable. Moreover, the consistency conditions given in Definitions 4 and 5 are based on the notion of certainty and they are too strict to reflect a real decision case. In addition, when interval numbers, triangular fuzzy numbers and trapezoidal fuzzy numbers are applied to evaluate the opinions of decision makers, the consistency of the corresponding preference matrices is mainly addressed by using some crisp preference matrices. The given methods seem to have too much mathematical techniques and too little real meanings in a decision making problem.

On the other hand, under the assumption of rational economics, it is natural that the judgements are consistent, acceptably consistent or transitive. However, as pointed out in [10], the consistency, acceptable consistency and transitivity of the judgements even together with the rational are not required in the AHP. The reason may be based on that the consistency degree of the judgements can be revealed at the end of the decision making process. Then the question is existing that how one will choose the best alternative in disordered alternatives. Hence we consider that the basic requirement of transitivity of the judgements is requisite, because the decision maker is rational to some extent in the most cases. In our opinions, to model a perfectly rational behaviour, the consistency analysis of the judgements in the typical AHP is necessary. When the fuzzy set theory is applied to the AHP, the behaviour of decision makers are only considered to be rational to some extent, so the weakly transitive relations of the judgements should be considered. It is unsuitable to simply apply the typical consistency to preference relations with fuzzy numbers. Recently, the new concept of approximation-consistency of interval multiplicative reciprocal matrices has been proposed by considering that the preference relations with fuzzy numbers are inconsistent in essence ${ }^{[61,62]}$. Moreover, some important properties of the typical consistency such as the invariance with respect to the permutations of alternatives may be not satisfied for a weak consistency. In the future, the weak consistency of prefer- 
ence relations with interval numbers, triangular numbers, trapezoidal fuzzy numbers should be studied comprehensively.

In the end, with respect to multiplicative reciprocal preference relations in the typical AHP, we give the concept of fuzzy consistency. That is, we have the following definition:

Definition 28 Suppose that $\mathcal{A}=\left\{\boldsymbol{A}=\left(a_{i j}\right)_{n \times n} \mid a_{i j}>0, a_{i j} a_{j i}=1, \forall i, j, n \geq 2\right\}$ is a set of all multiplicative reciprocal matrices. The fuzzy consistency of $\boldsymbol{A}$ is defined by a mapping $\mathbb{F}$ such that

$$
\mathbb{F}: \mathcal{A} \rightarrow[0,1]
$$

When $\mathbb{F}(\boldsymbol{A})=0$, the comparison matrix $\boldsymbol{A}$ is completely inconsistent, and when $\mathbb{F}(\boldsymbol{A})=1$, the comparison matrix $\boldsymbol{A}$ is completely consistent.

The concept of fuzzy consistency of comparison matrices in the AHP is given under the consideration of the vagueness in the process of evaluating the judgements. When the decision maker evaluates her/his opinions on alternatives and gives a comparison matrix, there exists vagueness about the consistency of judgments due to the complexity of the considered problem, the limitation of thinking and the incomplete of information. That is, for a perfect case, the expert may give a consistent and convincing comparison matrix, and for a fully irrational case, the expert may give a disordered and unconvincing comparison matrix. Generally, under the assumption of limited rational economics, the expert would give a comparison matrix with consistency to some extent. Or rather, it is reasonable and rational that the given comparison matrix owns the transitivity and some deviation from consistency. In order to capture the vagueness, we give the concept of fuzzy consistency in Definition 28. According to Definition 28, all the inconsistency indexes of comparison matrices should be normalized if they are considered the solid forms of fuzzy consistency. As compared to the axiomatic properties of inconsistency indices ${ }^{[16]}$, the concept of fuzzy consistency is a fuzzifying method for characterizing the inconsistency of the judgements. Definition 28 will be extended to preference relations with fuzzy numbers and applied in the decision analysis. Furthermore, it should be verified by using the modern theory of subjective measurement in the future.

\section{Conclusions}

The underlying idea and methods for capturing the consistency and transitivity of comparison matrices in the AHP and fuzzy AHP have been reviewed. It is concluded that there is a substantial controversy in analyzing the consistency of preference matrices with interval numbers and triangular fuzzy numbers, which should be clarified in the future. Under the assumption of rational behavior, the consistency analysis of the judgements by using the typical AHP should be necessary. When the fuzzy set theory is applied to the AHP, the weak consistency of preference relations with interval numbers, triangular numbers and trapezoidal fuzzy numbers should be studied comprehensively. One of the reasons is that the requirement of the typical consistency is incompatible with the idea of fuzzy set theory in modelling vagueness. It is concluded that the preference relations with fuzzy numbers are inconsistent in essence. Some important properties of the typical consistency may be not satisfied for the preference relations with fuzzy numbers such as the invariance with respect to the permutations of alternatives. Under the consideration of the vagueness in the process of evaluating the judgements, 
the concept of fuzzy consistency of comparison matrices in the AHP is given. We will study the applications of fuzzy consistency of preference relations and verify the given concept in terms of the modern theory of subjective measurement. In group decision making, the consistency of the group depends on the consistency level of each individual decision maker. It is important to deal with the case that an individual decision maker is extremely contrary to the group. Group decision making methods in terms of fuzzy AHP will be future proposed when the existing controversies are clarified and new weak-consistency concepts of preference relations with fuzzy numbers will be given.

\section{References}

[1] Saaty T L. A scaling method for priorities in hierarchical structures. Journal of Mathematical Psychology, 1977, 15(3): 234-281.

[2] Saaty T L. The analytic hierarchy process. McGraw-Hill, New York, 1980.

[3] Xu Z S, Wei C P. A consistency improving method in the analytic hierarchy process. European Journal of Operational Research, 1999, 116(2): 443-449.

[4] Bellman R E, Zadeh L A. Decision-making in a fuzzy environment. Management Science, 1970, 17(4): B-141-B-164.

[5] Zadeh L A. Fuzzy sets. Information Control, 1965, 8(3): 338-353.

[6] Pedrycz W. Why triangular membership functions? Fuzzy Sets and Systems, 1994, 64(1): 21-30.

[7] Van Laarhoven P J M, Pedrycz W. A fuzzy extension of Saaty's priority theory. Fuzzy Sets and Systems, 1983, 11(1-3): 229-241.

[8] Saaty T L, Vargas L G. Uncertainty and rank order in the analytic hierarchy process. European Journal of Operational Research, 1987, 32(1): 107-117.

[9] Buckley J J. Fuzzy hierarchical analysis. Fuzzy Sets and Systems, 1985, 17(3): 233-247.

[10] Saaty T L. Axiomatic foundation of the analytic hierarchy process. Management Science, 1986, 32(7): $841-855$.

[11] Black D. On the rationale of group decision-making. Journal of Political Economy, 1948, 56(1): 23-34.

[12] Crawford G. The geometric mean procedure for estimating the scale of a judgment matrix. Mathematical Modelling, 1989, 9(3-5): 327-334.

[13] Peláez J I, Lamata M T. A new measure of consistency for positive reciprocal matrices. Computers \& Mathematics with Applications, 2003, 46(12): 1839-1845.

[14] Stein W E, Mizzi P J. The harmonic consistency index for the analytic hierarchy process. European Journal of Operational Research, 2007, 177(1): 488-497.

[15] Brunelli M, Canal L, Fedrizzi M. Inconsistency indices for pairwise comparison matrices: A numerical study. Annals of Operations Research, 2013, 211(1): 493-509.

[16] Brunelli M, Fedrizzi M. Axiomatic properties of inconsistency indices for pairwise comparisons. Journal of the Operational Research Society, 2015, 66(1): 1-15.

[17] Brunelli M. Studying a set of properties of inconsistency indices for pairwise comparisons. Annals of Operations Research, 2017, 248(1): 143-161.

[18] Basile L, Dapuzzo L. Weak consistency and quasi-linear means imply the actual ranking. International Journal of Uncertainty, Fuzziness and Knowledge-Based Systems, 2002, 10(3): 227-239.

[19] Basile L, Dapuzzo L. Transitive matrices, strict preference order and ordinal evaluation operators. Soft Computing, 2006, 10(8): 933-940.

[20] Zimmermann H J. Fuzzy sets, decision making, and expert system. Kluwer Academic Publishers, Boston, 1987.

[21] Saaty T L, Vargas L G. Models, methods, concepts \& applications of the analytic hierarchy process (Second Edition). Springer Science+Business Media, New York, 2012.

[22] Orlovsky S A. Decision making with a fuzzy preference relation. Fuzzy Sets and Systems, 1978, 1(3): 155-167.

[23] Tanino T. Fuzzy preferene orderings in group decision making. Fuzzy Sets and Systems, 1984, 12(2): $117-131$. 
[24] Liu F, Zhang W G, Zhang L H. A group decision making model based on ageneralized ordered weighted geometric average operator with interval preference matrices. Fuzzy Sets and Systems, 2014, 246(7): 1-18.

[25] Herrera-Viedma F, Herrera F, Chiclana F, et al. Some issues on consistency of fuzzy preference relations. European Journal of Operational Research, 2004, 154(1): 98-109.

[26] Xu Z S. A survey of preference relations. International Journal of General Systems, 2007, 36(2): 179-203.

[27] Brunelli M. Introduction to the analytic hierarchy process. Springer, New York, 2015.

[28] Wang Y M, Yang J B, Xu D L. A two-stage logarithmic goal programming method for generating weights from interval comparison matrices. Fuzzy Sets and Systems, 2005, 152(3): 475-498.

[29] Dubois D. The role of fuzzy sets in decision sciences: Old techniques and new directions. Fuzzy Sets and Systems, 2011, 184(1): 3-28.

[30] Dubois D, Prade H. Fuzzy sets and systems: Theory and applications. Academic Press INC, NewYork, 1980.

[31] Liu F. Acceptable consistency analysis of interval reciprocal comparison matrices. Fuzzy Sets and Systems, 2009, 160(18): 2686-2700.

[32] Wang Z J. A note on "A goal programming model for incomplete interval multiplicative preference relations and its application in group decision-making". European Journal of Operational Research, 2015, 247(3): 867-871.

[33] Xia M M, Chen J. Studies on interval multiplicative preference relations and their application to group decision making. Group Decision and Negotiation, 2015, 24(1): 115-144.

[34] Xu Z S, Chen J. Some models for deriving the priority weights from interval fuzzy preference relations. European Journal of Operational Research, 2008, 184(1): 266-280.

[35] Liu F, Zhang W G, Fu J H. A new method of obtaining the priority weights from an interval fuzzy preference relation. Information Science, 2012, 185(1): 32-42.

[36] Wang Z J, Li K W. Goal programming approaches to deriving interval weights based on interval fuzzy preference relations. Information Science, 2012, 193(6): 180-198.

[37] Wang Z J, Li K W. A multi-step goal programming approach for group decision making with incomplete interval additive reciprocal comparison matrices. European Journal of Operational Research, 2015, 242(3): $890-900$.

[38] Wang T C, Chen Y H. Applying fuzzy linguistic preference relations to the improvement of consistency of fuzzy AHP. Information Science, 2008, 178(19): 3755-3765.

[39] Rezaei J, Ortt R, Scholten V. An improved fuzzy preference programming to evaluate entrepreneurship orientation. Applied Soft Computing, 2013, 13(5): 2749-2758.

[40] Liu F, Zhang W G, Zhang L H. Consistency analysis of triangular fuzzy reciprocal preference relations. European Journal of Operational Research, 2014, 235(3): 718-726.

[41] Wang Z J. Consistency analysis and priority derivation of triangular fuzzy preference relations based on modal value and geometric mean. Information Science, 2015, 314(9): 169-183.

[42] Xu Z S, Da Q L. An overview of operators for aggregating information. International Journal of Intelligent Systems, 2003, 18(9): 953-969.

[43] Saaty T L. Group decision making and the AHP. In The Analytic Hierarchy Process, Springer, Berlin, 1989: 59-67.

[44] Liu F, Zhang W G, Wang Z X. A goal programming model for incomplete interval multiplicative preference relations and its application in group decision-making. European Journal of Operational Research, 2012, 218(3): $747-754$.

[45] Xu Z S. On consistency of the weighted geometric mean complex judgement matrix in AHP. European Journal of Operational Research, 2000, 126(3): 683-687.

[46] Lin R, Lin J S J, Chang J. et al. Note on group consistency in analytic hierarchy process. European Journal of Operational Research, 2008, 190(3): 672-678.

[47] Yager R R. Families of OWA operators. Fuzzy Sets and Systems, 1993, 59(2): 125-148.

[48] Yager R R, Kacprzyk J. The ordered weighted averaging operators: Theory and Applications. Springer Science+Business Media, New York, 1997.

[49] Yager R R. On ordered weighted averaging aggregation operations in multicriteria decision making. IEEE Transactions on Systems, Man, Cybernetics — Part B: Cybernatics, 1988, 18(1): 183-190.

[50] Liu X W. Some properties of the weighted OWA operator. IEEE Transactions on Systems, Man, Cyber- 
netics — Part B: Cybernatics, 2006 36(1): 118-127.

[51] Yager R R, Filev D P. Induced ordered weighted averaging operators. IEEE Transactions on Systems, Man, Cybernetics — Part B: Cybernatics, 1999, 29(2): 141-150.

[52] Chiclana F, Herrera F, Herrera-Viedma E. Integrating three representation models in fuzzy multipurpose decision making based on fuzzy preference relations. Fuzzy Sets and Systems, 1998, 97(1): 33-48.

[53] Chiclana F, Herrera-Viedma E, Herrera F, et al. Some induced ordered weighted averaging operators and their use for solving group decision-making problems based on fuzzy preference relations. European Journal of Operational Research, 2007, 182(1): 383-399.

[54] Liu F, Zhang W G. TOPSIS-based consensus model for group decision-making with incomplete interval fuzzy preference relations. IEEE Transactions on Cybernetics, 2014, 44(8): 1283-1294.

[55] Herrera F, Herrera-Viedma E, Chiclana F. Multiperson decision-making based on multiplicative preference relations. European Journal of Operational Research, 2001, 129(2): 372-385.

[56] Xu Z S, Da Q L. The ordered weighted geometric averaging operators. International Journal of Intelligent Systems, 2002, 17(7): 709-716.

[57] Chiclana F, Herrera-Viedma E, Herrera F, et al. Induced ordered weighted geometric operators and their use in the aggregation of multiplicative preference relations. International Journal of Intelligent Systems, 2004, 19(3): 233-255.

[58] Liu F, Zhang W G, Shang Y F. A group decision-making model with interval multiplicative reciprocal matrices based on the geometric consistency index. Computers \& Industerial Engineering, 2016, 101(11): $184-193$.

[59] Saaty T L, Tran L T. On the invalidity of fuzzifying numerical judgments in the analytic hierarchy process. Mathematical and Computer Modelling, 2007, 46(7-8): 962-975.

[60] Ramík J, Korviny P. Inconsistency of pair-wise comparison matrix with fuzzy elements based on geometric mean. Fuzzy Sets and Systems, 2010, 161(11): 1604-1613.

[61] Liu F, Pedrycz W, Zhang W G. Limited rationality and its quantification through the interval number judgments with permutations. IEEE Transactions on Cybernetics, 2016, doi: 10.1109/TCYB.2016.2594491.

[62] Liu F, Pedrycz W, Wang Z X, et al. An axiomatic approach to approximation-consistency of triangular fuzzy reciprocal preference relations. Fuzzy Sets and Systems, 2017, doi: 10.1016/j.fss.2017.02.004. 